\title{
Exploring the role of hypercholesterolemia in tendon health and repair
}

\author{
Michael W. Hast ${ }^{1}$ \\ Joseph A. Abboud ${ }^{2}$ \\ Louis J. Soslowsky ${ }^{1}$ \\ 1 McKay Orthopaedic Research Laboratory, Universi- \\ ty of Pennsylvania, Philadelphia, PA, USA \\ 2 Rothman Institute, Thomas Jefferson University, \\ Philadelphia, PA, USA
}

\section{Corresponding author:}

Louis J. Soslowsky

McKay Orthopaedic Research Laboratory, Department of Orthopaedic Surgery, University of Pennsylvania

424a Stemmler Hall, 36th St. and Hamilton Walk, Philadelphia PA 19104-6081 USA

E-mail: soslowsk@upenn.edu

\section{Summary}

High cholesterol remains a significant healthcare problem, as more than $13 \%$ of adults in the U.S. are affected by hypercholesterolemia. The detrimental effects the disease has on cardiovascular health are well-documented, but the effects on the musculoskeletal system, and more specifically on tendons, have not been thoroughly examined. This paper provides an overview of work performed in our lab with various animal models to elucidate the relationship between high cholesterol and tendon biomechanical integrity and ability to heal. These studies highlight the complexity of relationships between multiple factors that influence tendon biomechanics, and it has offered a better understanding of the implications of high cholesterol on healthy and healing tendons.

KEY WORDS: animal model, high cholesterol, hypercholesterolemia, tendon.

\section{Introduction}

High cholesterol represents a significant healthcare problem both in the United States and throughout the world. More than $13 \%$ of adults in the U.S. are affected by hypercholesterolemia ${ }^{1}$, and the detri- mental effects the disease has on cardiovascular health have been well-documented ${ }^{2,3}$. In regard to tendon health, several clinical studies have demonstrated an association between familial hypercholesterolemia and Achilles tendon xanthomas and subsequent ruptures ${ }^{4,5}$. Our own clinical work has also suggested that there is a link between rotator cuff tears and high cholesterol in shoulder patients ${ }^{6}$. Despite these findings, the effects hypercholesterolemia on tendon properties has, until recently, not been studied.

Given the seemingly systemic nature of hypercholesterolemia, our group has sought to further assess the effect of high cholesterol in regard to the biomechanical integrity of tendons through the use of a series of IACUC-approved animal models that also meet the ethical standards of the Muscles, Ligaments and Tendons Journal ${ }^{7}$. We have been specifically interested in determining how high cholesterol impacts the mechanical properties of otherwise healthy tendons, and whether the disease impairs the tendon's ability to heal after acute injury. This manuscript reviews the work we have performed in regard to determining the effects of hypercholesterolemia on healthy tendons and tendon healing.

Summary and review of previously published studies

\section{Pig study}

Our first animal study focusing on hypercholesterolemia utilized a porcine model and consisted of seven male Yorkshire pigs (103 kg average) with a control group $(n=3)$ and a high cholesterol group $(n=4)$ that received a high cholesterol diet for 5 months ${ }^{8}$. At the end of the five month treatment, all animals were sacrificed and the previously described mechanical testing protocol was performed with a gauge length of $30 \mathrm{~mm}$ (Fig. 1). The specimen was subjected to a preload, preconditioning, and ramp to failure at $0.1 \% / \mathrm{s}$. Mean cholesterol levels at the time of sacrifice were $290 \mathrm{mg} / \mathrm{dL}$ for the hypercholesterolemic (HC) group and under $100 \mathrm{mg} / \mathrm{dL}$ for the control (CTL) group. No differences were noted in tendon size as measured by cross-sectional area, but biomechanical testing revealed significantly reduced stiffness $(p<0.002)$ and Young's modulus $(p<0.0001)$ in the $\mathrm{HC}$ group compared to CTL tendons. This finding was present for both tendon midsubstance and insertion site properties, and this was the first study re- 


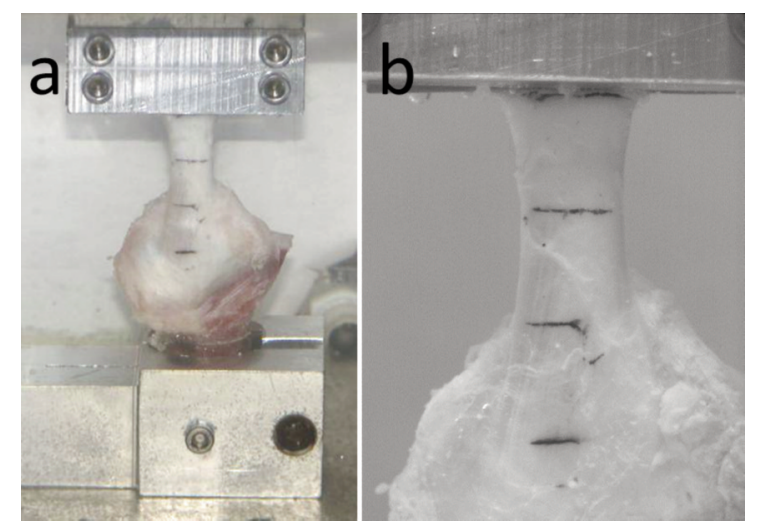

porting effects of hypercholesterolemia on native tendon mechanical properties. The finding of substantially reduced mechanics supported our clinical observations relating high cholesterol and the incidence of rotator cuff tendon tears ${ }^{6}$.

\section{Mouse studies}

We performed several subsequent studies that utilized a murine knock out model to examine the cumulative effects of hypercholesterolemia ${ }^{9-11}$. Based on the results of our porcine biceps investigation, we initially hypothesized that tendons from hypercholesterolemic mice would exhibit inferior baseline and healing mechanical properties compared to controls. The first study we would like to highlight consisted of forty male C57BL/6 CTL mice and 40 male C57BL/6 mice deficient for Apolipoprotein E (APOE) representing a hypercholesterolemic group ${ }^{11}$. To investigate
Figure 1. (a) Porcine biceps tendon placed in test fixture, submerged in a PBS bath at $37^{\circ} \mathrm{C}$, and (b) an example of an image captured during tensile testing ${ }^{8}$. the effects of an accumulation of exposure to high cholesterol, half of the animals were sacrificed at 14 weeks while the other animals were sacrificed at 40 weeks. Half of the animals in each group received a $0.75 \mathrm{~mm}$ punch biopsy defect to the central third of the left patellar tendon, while the right patellar tendon received a sham operation. The other half of the animals had no injury in order to serve as controls. Uninjured data showed no differences between groups in regards to cross-sectional area, maximum stress, or percent relaxation (Fig. 2). However 10-month 40week-old APOE mice showed a decrease in elastic modulus $(p<0.02)$, indicating a detrimental cumulative effect of hypercholesterolemia on tendon properties in this model. Surprisingly, tensile testing of patellar tendons from 14-week-old APOE mice receiving a unilateral full-thickness central defect resulted in normalized (comparing injured to contralateral sham) cross-sectional areas closer to baseline $(p<0.02)$ compared to controls.

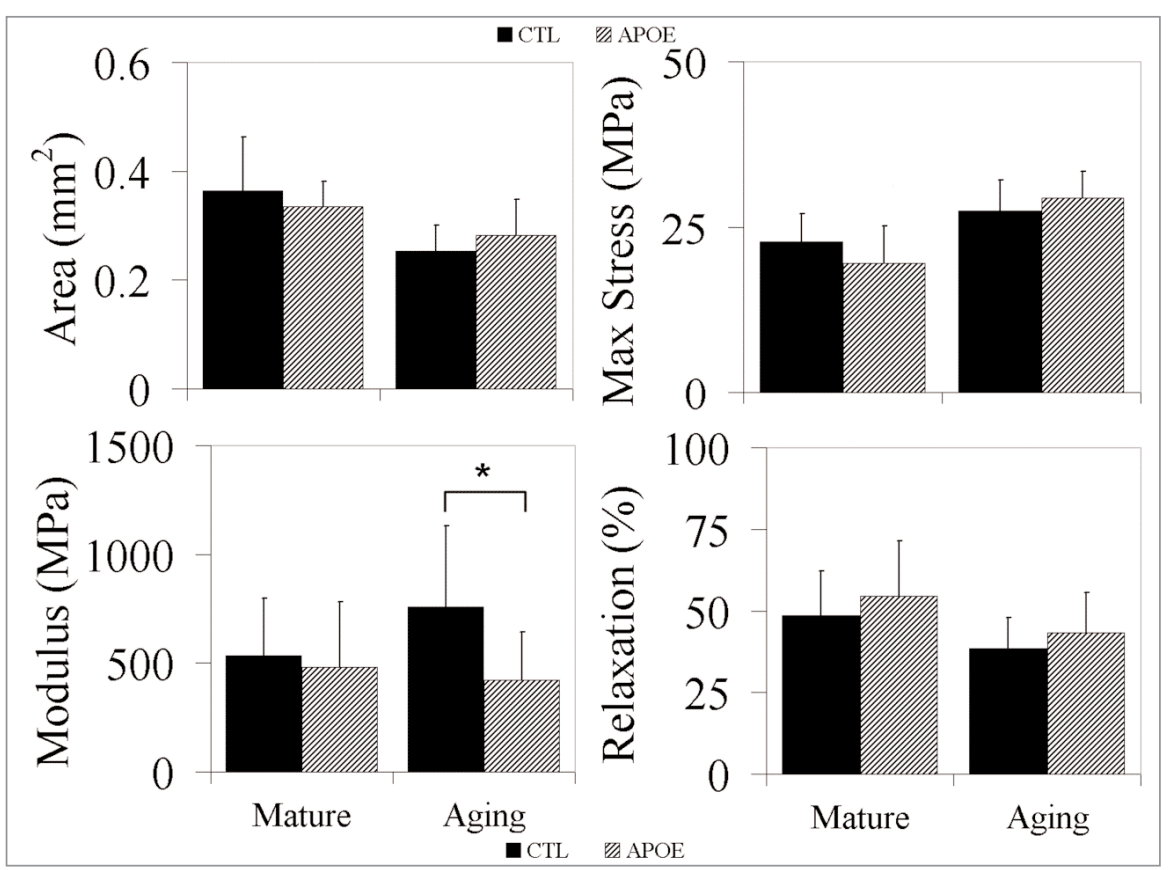

Figure 2. Biomechanical properties of tendon in uninjured CTL and APOE mice. Elevated cholesterol levels have a significant effect $(p=$ 0.02 ) on elastic modulus of the tendon in the aging group ${ }^{11}$. Reprinted with permission from the Journal of Orthopaedic Research. 
The objective of another study in knockout mice was to evaluate tendon healing in normal and hypercholesterolemic mice using our patellar tendon injury model ${ }^{10}$. We hypothesized that tendons from aging hypercholesterolemic mice would exhibit inferior baseline mechanical properties and tendon healing compared to normal controls. Twenty-four male C57BL/6 CTL mice and 24 male C57BL/6 mice APOE mice were used. For each group, ten animals were sacrificed without injury to provide baseline, uninjured data. Patellar tendons from the remaining 28 animals were injured as described previously. All animals were sacrificed at 43 weeks of age ( 3 weeks post-injury for operated animals). The tendon was then stamped into a dumbbell shape and cross-sectional area was again measured for use in calculation of material properties. Tensile testing progressed as follows: preload, preconditioning, stress relaxation for 10 minutes at $5 \%$ strain, return to gage length, and ramp to failure $(0.1 \% / \mathrm{s})$.

Again, uninjured patellar tendons from APOE mice showed a significant decrease in elastic modulus $(p=0.02)$ but this time we noted a trend toward increased cross-sectional area $(p=0.1)$ compared to control as well. It was found that normalized maximum stress was significantly lower in the APOE group than in the controls $(p=0.05)$ and there were no differences in normalized area or modulus. As hypothesized, APOE tendons exhibited reduced healing strength and baseline elastic modulus compared to controls. The results from this second knock out study are contrary to our initial finding with 14-weekold mice using similar methods ${ }^{9}$. It should be noted that the mice in the current study were appreciably older than in the former study. The reduction in tendon healing in aging hypercholesterolemic tendons may be linked to the cumulative effects of intratendinous cholesterol deposition or relative tissue ischemia due to vascular compromise, as seen clinically in older patients. Although the results of these knock out studies are certainly intriguing, it must be noted that they were in genetically altered mice, and the implications of these results may be limited by potential compensatory mechanisms associated with such an approach. Additionally, the knockout mouse model used in this approach is more comparable to the less-common condition of familial hypercholesterolemia seen in humans and does not clinically replicate the condition of mixed dyslipidemia seen by most patients suffering from hypercholesterolemia. Therefore, further work was performed to address the translational potential of this research direction.

\section{Rat studies}

To further model the human condition of diet-induced high cholesterol, we studied the effects of diet-induced hypercholesterolemia on rotator cuff tendon mechanics in a rat model ${ }^{12,13}$. The objective of our first study utilizing this model system was to evaluate tendon mechanical properties in our existing rat rota- tor cuff model following three months of exposure to two high-cholesterol diets ${ }^{12}$. We formulated two high cholesterol diets and hypothesized that both would induce hypercholesterolemia in the rats. We further hypothesized that supraspinatus tendons from hypercholesterolemic rats would exhibit reduced mechanical properties compared to rats fed a normal diet. Thirty male Sprague-Dawley rats (400-450 grams) were used in this study, with ten rats receiving a high-cholesterol diet consisting of $4 \%$ cholesterol and $1 \%$ sodium cholate (HC1 group) another group of ten rats receiving a different diet formulation consisting of $2 \%$ cholesterol (HC2 group), and the remaining ten rats receiving standard chow to serve as controls (CTL group).

Following sacrifice, lipid analysis confirmed that both high-cholesterol diets produced increased TC levels as well as TC:HDL ratios. Rats in the $\mathrm{HC} 2$ group also demonstrated a decline in HDL levels. Triglycerides were decreased in the $\mathrm{HC} 1$ rats. Biomechanical testing of supraspinatus tendons showed consistent increases in stiffness and elastic modulus in both high cholesterol rat groups. The findings of increased stiffness and modulus in hypercholesterolemic rats were in direct contrast to the previous results found in the previously discussed porcine (biceps) and murine (patellar) experiments. This may be due to the differences in type, location, and function of the different tendons and how these relate to various intrinsic and extrinsic factors. While both three-month diet courses did produce marked increases in cholesterol as measured in the blood, this time frame may not have been long enough for the deleterious cumulative effects of hypercholesterolemia seen in our previous work.

The objective of our second study involving rats on a $\mathrm{HC}$ diet was to evaluate the time course of healing of supraspinatus tendons in our rat rotator cuff injury model $^{13}$. Sixty-four Sprague Dawley rats (400-450 g) were used in this study, with 32 of the animals receiving a high cholesterol diet over the course of 6 months. All animals were subjected to a unilateral supraspinatus detachment and repair surgery, with contralateral limbs serving as within-animal comparative data. Animals continued their respective diet courses, and their supraspinatus tendons were biomechanically or histologically evaluated at 2,4 , and 8 weeks postoperatively. Biomechanical testing revealed a significant reduction in normalized stiffness in hypercholesterolemic rats compared with controls at 4 weeks after injury (Fig. 3), whereas histologic analyses showed no significant differences in collagen organization, cellularity, or cell shape between groups.

\section{Animal model comparisons}

Given the conflicting nature of the results we have discovered, we also sought to assess the utility of small versus large animal model systems for translational studies by exploring the effect of hypercholes- 


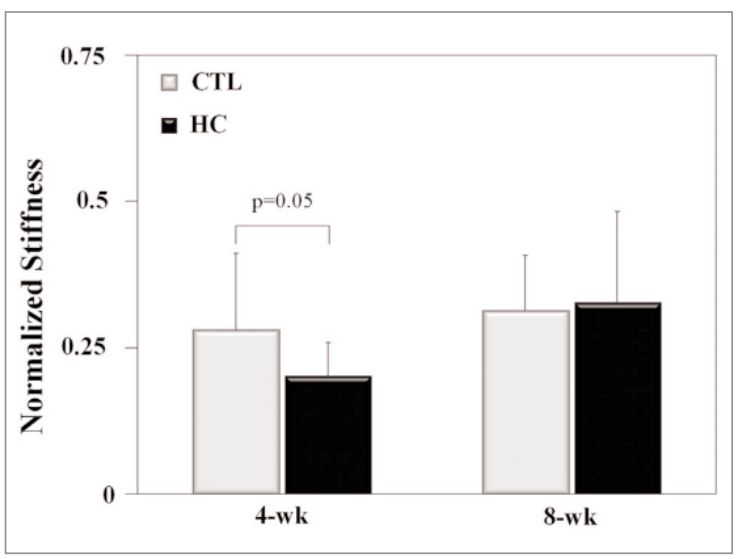

Figure 3. Normalized stiffness for the repaired rat supraspinatus with $\mathrm{HC}$ was lower $(p=0.05)$ than the control at 4 weeks, but not at 8 weeks ${ }^{13}$. Reprinted with permission from the Journal of Shoulder and Elbow Surgery.

terolemia on supraspinatus tendon elastic mechanical properties in mice, rats, and monkeys ${ }^{14}$. We hypothesized that stiffness and elastic modulus would be increased in tendons across species due to hypercholesterolemia. Supraspinatus tendons from normal and $\mathrm{HC}$ mice, rats, and monkeys were used in this study. Cholesterol levels were manipulated for the mice through the previously described knock out model, and hypercholesterolemia for the rat and monkey models was investigated through changes in diet. After sacrifice and dissection, tendons from the different species underwent the mechanical protocol previously described.

Overall, $\mathrm{HC}$ animals had significantly altered plasma lipid profiles. Biomechanical testing showed a significant increase in stiffness compared with control in HC mice and rats, as well as a nonsignificant trend for $\mathrm{HC}$ monkeys. Elastic modulus was also significantly increased in $\mathrm{HC}$ mice and monkeys, with $\mathrm{HC}$ rats showing a trend (Fig. 4). There was a strong consistency across species and between small and large animals. This was a bit surprising, as the aged mice were exposed to lifelong hypercholesterolemia while the rats and nonhuman primates only experienced increased cholesterol levels once they began their high-cholesterol diets. This suggests that these increased mechanical properties may be inherent to the effect of hypercholesterolemia on supraspinatus tendon rather than due to an effect of cumulative exposure time to the effects of $\mathrm{HC}$.

\section{Conclusions}

Although many authors have theorized that tendon disease is multifactorial, relevant factors must be evaluated one by one in a systematic manner with the goal of reducing the impact of such costly musculoskeletal problems. The information gained from these ongoing studies will continue to affect the field of tendon biology and biomechanics through the fur-

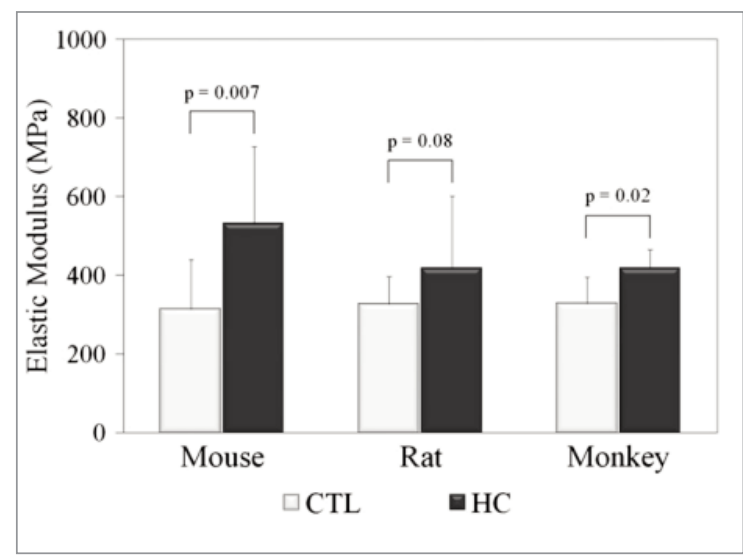

Figure 4. Moduli were increased in $\mathrm{HC}$ tendons compared with CTL across species ${ }^{14}$. Reprinted with permission from the Journal of Shoulder and Elbow Surgery.

ther identification of intrinsic risk factors for tendon disease. The studies have provided a better understanding of the implications of mixed hyperlipidemia on the long term structure and function of tendons. If a mechanistic cause of hypercholesterolemia induced tendon disease is identified, then potential therapeutic interventions such as the use of pharmacotherapy may be targeted to help combat this problem.

\section{References}

1. National Center for Health Statistics. Health, United States, 2012: With Special Feature on Emergency Care. Hyattsville, MD; 2013.

2. Langer T, Strober W, Levy RI. The metabolism of low density lipoprotein in familial type II hyperlipoproteinemia. J Clin Invest. 1972;51(6):1528-1536.

3. Mensink RP, Katan MB. Effect of Dietary trans Fatty Acids on High-Density and Low-Density Lipoprotein Cholesterol Levels in Healthy Subjects. N Engl J Med. 1990;323(7):439445.

4. Mathiak G, Wening JV, Mathiak M, Neville LF, Jungbluth K$\mathrm{H}$. Serum cholesterol is elevated in patients with Achilles tendon ruptures. Arch Orthop Trauma Surg. 1999;119(5-6):280284.

5. Ozgurtas T, Yildiz C, Serdar M, Atesalp S, Kutluay T. Is high concentration of serum lipids a risk factor for Achilles tendon rupture? Clin Chim Acta. 2003;331(1-2):25-28.

6. Abboud JA, Kim JS. The Effect of Hypercholesterolemia on Rotator Cuff Disease. Clin Orthop Relat Res. 2010;468(6):14931497.

7. Padulo J, Oliva F, Frizziero A, Maffulli N. Muscles, Ligaments and Tendons Journal. Basic principles and recommendations in clinical and field science research. MLTJ. 2013;4: 250-252.

8. Beason D, Kuntz A, Hamamdzic R, Mohler E, Abboud J. High Cholesterol Adversely Affects Biceps Tendon Mechanical Properties in a Porcine Model. Trans Orthop Res Soc. 2009:184.

9. Beason D, Abboud J, Bassora R, Soslowsky L. Tendon Healing in a Mouse Injury Model: The Role of Hypercholesterolemia. Trans Orthop Res Soc. 2008: 814.

10. Beason D, Abboud J, Bassora A, Kuntz A, Soslowsky L. Hy- 
percholesterolemia is Detrimental to Tendon Properties and Healing in a Mouse Injury Model. Trans Orthop Res Soc. 2009:1418.

11. Beason DP, Abboud JA, Kuntz AF, Bassora R, Soslowsky LJ. Cumulative effects of hypercholesterolemia on tendon biomechanics in a mouse model. J Orthop Res Off Publ Orthop Res Soc. 2011;29(3):380-383.

12. Beason D, Hsu J, Edelstein L, et al. Effect of Diet-Induced Hypercholesterolemia on Rotator Cuff Tendon Mechanics in a
Rat Model. Trans Orthop Res Soc. 2011:223.

13. Beason DP, Tucker JJ, Lee CS, Edelstein L, Abboud JA Soslowsky LJ. Rat rotator cuff tendon-to-bone healing properties are adversely affected by hypercholesterolemia. J Shoulder Elb Surg Am Shoulder Elb Surg Al. 2013.

14. Beason DP, Hsu JE, Marshall SM, et al. Hypercholesterolemia increases supraspinatus tendon stiffness and elastic modulus across multiple species. J Shoulder Elbow Surg. 2013;22(5):681-686 . 